NASA/TM-2003-212646

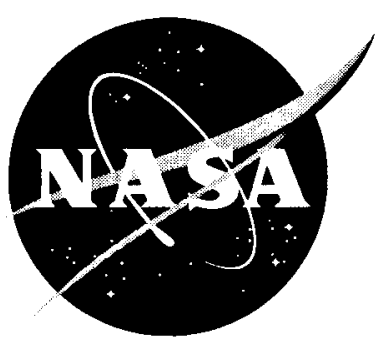

\title{
Continuous-Time Bilinear System Identification
}

\author{
Jer-Nan Juang
}

Langley Research Center, Hampton, Virginia 
Since its founding, NASA has been dedicated to the advancement of aeronautics and space science. The NASA Scientific and Technical Information (STI) Program Office plays a key part in helping NASA maintain this important role.

The NASA STI Program Office is operated by Langley Research Center, the lead center for NASA's scientific and technical information. The NASA STI Program Office provides access to the NASA STI Database, the largest collection of aeronautical and space science STI in the world. The Program Office is also NASA's institutional mechanism for disseminating the results of its research and development activities. These results are published by NASA in the NASA STI Report Series, which includes the following report types:

- TECHNICAL PUBLICATION. Reports of completed research or a major significant phase of research that present the results of NASA programs and include extensive data or theoretical analysis. Includes compilations of significant scientific and technical data and information deemed to be of continuing reference value. NASA counterpart of peerreviewed formal professional papers, but having less stringent limitations on manuscript length and extent of graphic presentations.

- TECHNICAL MEMORANDUM. Scientific and technical findings that are preliminary or of specialized interest, e.g., quick release reports, working papers, and bibliographies that contain minimal annotation. Does not contain extensive analysis.

- CONTRACTOR REPORT. Scientific and technical findings by NASA-sponsored contractors and grantees.
- CONFERENCE PUBLICATION. Collected papers from scientific and technical conferences, symposia, seminars, or other meetings sponsored or co-sponsored by NASA.

- SPECIAL PUBliCATION. Scientific, technical, or historical information from NASA programs, projects, and missions, often concerned with subjects having substantial public interest.

- TECHNICAL TRANSLATION. Englishlanguage translations of foreign scientific and technical material pertinent to NASA's mission.

Specialized services that complement the STI Program Office's diverse offerings include creating custom thesauri, building customized databases, organizing and publishing research results ... even providing videos.

For more information about the NASA STI Program Office, see the following:

- Access the NASA STI Program Home Page at http://www.sti.nasa.gov

- E-mail your question via the Internet to help@sti.nasa.gov

- Fax your question to the NASA STI Help Desk at (301) 621-0134

- Phone the NASA STI Help Desk at (301) 621-0390

- Write to:

NASA STI Help Desk

NASA Center for AeroSpace Information 7121 Standard Drive Hanover, MD 21076-1320 
NASA/TM-2003-212646

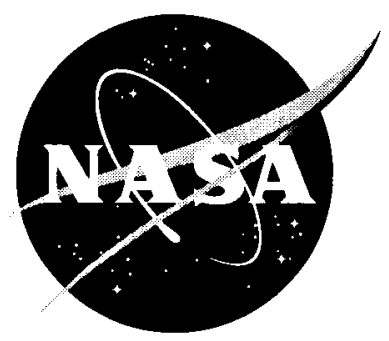

\section{Continuous-Time Bilinear System Identification}

Jer-Nan Juang

Langley Research Center, Hampton, Virginia 
Available from:

NASA Center for AeroSpace Information (CASI)

7121 Standard Drive

Hanover, MD 21076-1320

(301) 621-0390
National Technical Information Service (NTIS) 5285 Port Royal Road Springfield, VA 22161-2171 (703) 605-6000 


\title{
Continuous-Time Bilinear System Identification
}

\author{
Jer-Nan Juang \\ Structural Dynamics Branch \\ NASA Langley Research Center \\ Hampton, VA 23681
}

\begin{abstract}
The objective of this paper is to describe a new method for identification of a continuous-time multi-input and multi-output bilinear system. The approach is to make judicious use of the linear-model properties of the bilinear system when subjected to a constant input. Two steps are required in the identification process. The first step is to use a set of pulse responses resulting from a constant input of one sample period to identify the state matrix, the output matrix, and the direct transmission matrix. The second step is to use another set of pulse responses with the same constant input over multiple sample periods to identify the input matrix and the coefficient matrices associated with the coupling terms between the state and the inputs. Numerical examples are given to illustrate the concept and the computational algorithm for the identification method.
\end{abstract}

\section{Introduction}

System identification is a methodology used to characterize a dynamical or other engineering system with measurements of the input-output signals. Mathematicians and engineers have developed a number of approaches to address the identification problem. The identification of a linear time-invariant system is relatively well understood and theoretically well developed $[1,2]$. This is not true for the identification of a nonlinear system, although some progress has been made in the identification of nonlinear systems over the past few decades [3-19].

There is a class of nonlinear systems called bilinear systems whose dynamics are jointly linear in the state and the force variables. It is a simple nonlinear extension of a 
linear system. The concept of bilinear systems was introduced in the 1960's (see the surveys of Refs. [5] and [6]). References [7] and [8] provide a survey of bilinear-related system-theory methods and their contributions to problems such as stabilization, controllability, and observability. Bilinear systems have been studied extensively and applied successfully to several problems [15]. Recently, research activities in identification of bilinear systems have been focused on the so-called "discrete-time" model identification [19]. The discrete-time model is an approximation obtained by linearizing the continuous one with a method such as the finite difference. In contrast, we focus on the identification of a continuous-time bilinear system without any approximation.

A new method is introduced in this paper for identification of a continuous-time multi-input and multioutput bilinear system. When the input of a bilinear system is a constant, the bilinear system becomes a linear system. This special characteristic is the basis for the identification method. Two steps are required for the identification process. The first step begins with generating a set of pulse responses with a constant input applied one at a time over one sample period. The pulse responses are then used to form a Hankel matrix consisting of system Markov parameters to identify the state matrix, the output matrix, and the direct transmission matrix. The identification step is quite similar, if not identical, to the identification of a linear system $[1,2]$. This step establishes a specific set of coordinates for the whole identification process. This set of coordinates is not unique, depending mainly on the size of Hankel matrix and the resulting choice of matrix that represents the observability matrix. The second step starts by generating another set of pulse responses with the same constant input as the frst step but for multiple sample periods. These multiple-pulse responses are used to define another set of Markov parameters to form a Hankel-like matrix for each input. The observability matrix obtained in the first step is then applied to the Hankellike matrix to compute the corresponding controllability matrix of the input to identify the input vector and the coefficient matrix associated with the coupling terms between the state and the input.

Simple examples are given to demonstrate how to apply the method to identify a continuous-time bilinear system and how to transfer the identified model from one set of 
coordinates to the other set of coordinates. The coordinate transformation also serves as a way of verifying the identified system.

\section{Basic Formulation}

Let $x$ and $A_{c}$, be the state vector of dimension $n \times 1$ and its corresponding state matrix of $n \times n$, and $u$ and $B_{c}$ be the input vector of $r \times 1$ and its corresponding input matrix of $n \times r$. Subscript $c$ signifies the associated quantity in the continuous-time domain. The bilinear state equation in the continuous-time domain is commonly expressed by

$$
\dot{x}=A_{c} x+B_{c} u+\sum_{i=1}^{r} N_{c i} x u_{i}
$$

where the coupling term $x u_{i}$ between the state vector $x$ and each individual $u_{i}(i=1, \ldots, r)$ in the input vector $u$ is weighted by the matrix $N_{c i}$ of $n \times n$. The measurement equation is identical to the one for a linear system that is commonly described by

$$
y=C x+D u
$$

where $\mathrm{y}$ is the output measurement vector of $m \times 1, C$ is the output matrix of $m \times n$ and $D$ is the direct transmission matrix of $m \times r$.

For simplicity, consider only one input at a time. Equation (1) reduces to

$$
\dot{x}=A_{c} x+b_{c i} u_{i}+N_{c i} x u_{i}
$$

where $b_{c i}$ is the $i$ th column of $B_{\mathrm{c}}$ associated with the input $u_{\mathrm{k}}$. Assuming $u_{i}=\mathrm{v}_{i}$ where $v_{i}$ is a pre-specified constant, the continuous-time state equation (3) further reduces to

$$
\dot{x}=\left(A_{c}+\mathrm{v}_{i} N_{c i}\right) x+b_{c i} \mathrm{v}_{i}
$$

The discrete-time model of this system is

$$
x(k+1)=\bar{A}_{i} x(k)+\bar{b}_{i} ; \quad i=1,2, \ldots, r
$$

with the measurement equation

$$
y_{i}(k)=C x(k)+\bar{d}_{i}
$$

where $d_{\mathrm{i}}$ is the $i$ th column of $D$ associated with the input $u_{\mathrm{k}}$ and

$$
\begin{aligned}
& \bar{A}_{i}=e^{\left(A+v_{i} N_{c i}\right) \Delta t} \\
& \bar{b}_{i}=\int_{0}^{\Delta} e^{\left(A+v_{i} N_{c i}\right) \tau} d \tau b_{c i} v_{i}
\end{aligned}
$$




$$
\bar{d}_{i}=d_{i} v_{i}
$$

The quantity $\Delta t$ is the time interval for data sampling. Assuming that the initial state $x(0)$ is a zero vector, i.e., $x(0)=0_{n \times 1}$, the state response for the discrete-time model, Eq. (5), can be computed by:

$$
\begin{aligned}
x(0) & =0_{n \times 1} \\
x(1) & =\bar{A}_{i} x(0)+\bar{b}_{i}=\bar{b}_{i} \\
x(2) & =\bar{A}_{i}^{2} x(1)+\bar{b}_{i}=\bar{A}_{i} \bar{b}_{i}+\bar{b}_{i} \\
x(3) & =\bar{A}_{i}^{3} x(2)+\bar{b}_{i}=\bar{A}_{i}^{2} \bar{b}_{i}+\bar{A}_{i} \bar{b}_{i}+\bar{b}_{k} \\
& \vdots \\
x(N) & =\bar{A}_{i}^{N} x(1)+\bar{b}_{i}=\bar{A}_{i}^{N-1} \bar{b}_{i}+\bar{A}_{i}^{N-2} \bar{b}_{i}+\cdots+\bar{A}_{i} \bar{b}_{i}+\bar{b}_{i}
\end{aligned}
$$

After $t>N \Delta t$, let $u_{i}(t)=0$. The state equation (3) reduces to the simple form

$$
\dot{x}=A_{c} x
$$

Its discrete-time model is

$$
x(k+1)=A x(k)
$$

where

$$
A=e^{A \Delta t}
$$

The free decay response after $t>N \Delta t$ becomes

$$
\begin{gathered}
x(N+1)=A x(k)=A\left(\bar{A}_{i}^{N-1} \bar{b}_{i}+\bar{A}_{i}^{N-2} \bar{b}_{i}+\cdots+\bar{A}_{i} \bar{b}_{i}+\bar{b}_{i}\right) \\
x(N+2)=A^{2} x(k)=A^{2}\left(\bar{A}^{N-1} \bar{b}_{i}+\bar{A}_{i}^{N-2} \bar{b}_{i}+\cdots+\bar{A}_{i} \bar{b}_{i}+\bar{b}_{i}\right) \\
\vdots \\
x(N+\ell)=A^{\prime} x(k)=A^{l}\left(\bar{A}^{N-1} \bar{b}_{i}+\bar{A}_{i}^{N-2} \bar{b}_{i}+\cdots+\bar{A}_{i} \bar{b}_{i}+\bar{b}_{i}\right)
\end{gathered}
$$

where $\ell$ is an integer indicating the data length of the free-decay response.

From Eqs. (2), (10), and (14), the measurement quantities $y_{i}(k)$ for $k=0,1, \cdots, N+\ell$ due to the force excitation of $u_{k}=v_{k}$ can thus be computed as 


$$
\begin{aligned}
y_{i}(0) & =C x(0)+\bar{d}_{i}=\bar{d}_{i} \\
y_{i}(1) & =C x(1)+\bar{d}_{i}=C \bar{b}_{i}+\bar{d}_{i} \\
y_{i}(2) & =C x(2)+\bar{d}_{i}=C\left(\bar{A}_{i} \bar{b}_{i}+\bar{b}_{i}\right)+\bar{d}_{i} \\
& \vdots \\
y_{i}(N) & =C x(N)=C\left(\bar{A}_{i}^{N-1} \bar{b}_{i}+\bar{A}_{i}^{N-2} \bar{b}_{i}+\cdots+\bar{A}_{i} \bar{b}_{i}+\bar{b}_{i}\right) \\
y_{i}(N+1) & =C x(N+1)=C A\left(\bar{A}_{i}^{N-1} \bar{b}_{i}+\bar{A}_{i}^{N-2} \bar{b}_{i}+\cdots+\bar{A}_{i} \bar{b}_{i}+\bar{b}_{i}\right) \\
& \vdots \\
y_{i}(N+\ell) & =C x(N+C)=C A^{\prime}\left(\bar{A}_{i}^{N-1} \bar{b}_{i}+\bar{A}_{i}^{N-2} \bar{b}_{i}+\cdots+\bar{A}_{i} \bar{b}_{i}+\bar{b}_{i}\right)
\end{aligned}
$$

The upper portion, $y(0), y(1), \cdots, y(N)$, of Eq. (15), corresponds to the multiple-pulse response resulting from a constant force over multiple sample periods. But the lower portion, $y(N+1), y(N+2), \cdots, y(N+\ell)$, corresponds to the free-decay response.

It is clear that the system matrices/vectors, $A_{c}, b_{c i}, N_{c i}, C, d_{i}$, are embedded in the output quantities shown in Eq. (15). First of all, we need to use the output measurements to extract the discrete-time matrices/vectors, $A, C, \bar{A}, \bar{b}_{i}, d_{i}$. It is worth to stress that the multiple-pulse response and the free-decay response result from two different discrete models.

The free-decay response, $y(N+1), y(N+2), \cdots, y(N+\ell)$, after $t>N \Delta t$ is quite similar, if not identical, to the pulse response for a linear system. Any linear system identification technique may be applied to compute the state matrix $A$ and the output matrix $C$. The key idea is to make judicious use of this linear portion of the bilinear system. The identification problems for linear systems have been extensively studied and many good techniques have been developed and implemented.

\section{System Identification Method}

The identification method requires two steps. The first step is to identify the state matrix $A_{\mathfrak{c}}$, the output matrix $C$, and the data transmission matrix $D$. The second step is to determine the input matrices $B_{\mathrm{c}}$, and $N_{\mathrm{i}}$ for the coupling term between the state vector $\mathrm{x}$ and the $i$ th input $u_{\mathrm{i}}$. 


\section{Identification of $A_{c}, C$, and $D$ :}

First, let us apply a pulse of magnitude $v_{1}$ to the system for one time step $\Delta t$ to generate the pulse response for the first input $u_{1}$. From Eq. (15) for $N=1$, the pulse response has the following expression.

$$
\begin{gathered}
y_{1}(0)=\bar{d}_{1} \\
y_{1}(1)=C \bar{b}_{1} \\
y_{1}(2)=C A \bar{b}_{1} \\
\vdots \\
y_{1}(\ell+1)=C A \bar{b}_{1}
\end{gathered}
$$

All other input pulse responses can be similarly generated to yield

$$
\begin{array}{ccc}
y_{2}(0)=\bar{d}_{2} & \cdots & y_{r}(0)=\bar{d}_{r} \\
y_{2}(1)=C \bar{b}_{2} & \cdots & y_{r}(1)=C \bar{b}_{r} \\
y_{2}(2)=C A \bar{b}_{2} & \cdots & y_{r}(2)=C A \bar{b}_{r} \\
\vdots & \vdots & \vdots \\
y_{2}(\ell+1)=C A^{\prime} \bar{b}_{2} & \cdots & y_{r}(\ell+1)=C A^{\prime} \bar{b}_{r} .
\end{array}
$$

Equation (17) is obtained by replacing the subscript 1 representing the first input with the other input integers 2 through $r$. Let us define the system Markov parameters to be

$$
\begin{aligned}
& Y_{1}(0)=\left[\begin{array}{llll}
y_{1}(0) & y_{2}(0) & \cdots & y_{r}(0)
\end{array}\right]=\left[\begin{array}{llll}
\bar{d}_{1} & \bar{d}_{2} & \cdots & \bar{d}_{r}
\end{array}\right]=\bar{D} \\
& Y_{1}(1)=\left[\begin{array}{llll}
y_{1}(1) & y_{2}(1) & \cdots & y_{r}(1)
\end{array}\right]=\left[\begin{array}{llll}
C \bar{b}_{1} & C \bar{b}_{2} & \cdots & C \bar{b}_{r}
\end{array}\right] \\
& Y_{1}(2)=\left[\begin{array}{llll}
y_{1}(2) & y_{2}(2) & \cdots & y_{r}(2)
\end{array}\right]=\left[\begin{array}{llll}
C A \bar{b}_{1} & C A \bar{b}_{2} & \cdots & C A \bar{b}_{r}
\end{array}\right] \\
& Y_{1}(\ell+1)=\left[\begin{array}{llll}
y_{1}(\ell+1) & y_{2}(\ell+1) & \cdots & y_{r}(\ell+1)
\end{array}\right]=\left[\begin{array}{lllll}
C A^{\prime} \bar{b}_{1} & C A^{\prime} \bar{b}_{2} & \cdots & C A \bar{b}_{r}
\end{array}\right]
\end{aligned}
$$

The use of the subscript 1 for $Y_{1}(k)(k=1,2, \ldots, \ell+1)$ is intended to signify one-time-step pulse response. Equation (18) provides the basic parameters for system identification. Indeed, let us form a Hankel matrix as follows.

$$
H_{1}=\left[\begin{array}{cccc}
Y_{1}(1) & Y_{1}(2) & \cdots & Y_{1}(\beta) \\
Y_{1}(2) & Y_{1}(3) & \cdots & Y_{1}(\beta+1) \\
\vdots & \vdots & \ddots & \vdots \\
Y_{1}(\alpha) & Y_{1}(\alpha+1) & \cdots & Y_{1}(\alpha+\beta-1)
\end{array}\right]=\left[\begin{array}{c}
C \\
C A \\
\vdots \\
C A^{\alpha-1}
\end{array}\right]\left[\begin{array}{llll}
\bar{B}_{1} & A \bar{B}_{1} & \cdots & A^{\beta-1} \bar{B}_{1}
\end{array}\right](1
$$

where 


$$
\bar{B}_{1}=\left[\begin{array}{llll}
\bar{b}_{1} & \bar{b}_{2} & \cdots & \bar{b}_{r}
\end{array}\right]
$$

The matrix product on the right-hand side of Eq. (19) shows the relationship between the system Markov parameters and the discrete-time system matrices. Obviously the Hankel matrix $H_{1}$ has the rank $n$ that is the order of the state matrix $A$ if we choose $\alpha$ and $\beta$ such that $\alpha m$ and $\beta r$ are larger than or equal to $n$ where $m$ is the number of outputs and $r$ is the number of inputs. Using the singular value decomposition (SVD) to decompose the Hankel matrix $H_{1}$ yields

$$
H_{1}=U_{1} \Sigma_{1} V_{1}^{T}
$$

where $\Sigma_{1}$ of $n \times n$ is a square matrix containing $n$ non-zero singular values. The matrix $U_{1}$ is of dimension $\alpha m \times n$ and the matrix $V_{1}$ is of dimension $\beta r \times n$.

From Eq. (19), one may choose

$$
U_{1}=\left[\begin{array}{c}
C \\
C A \\
\vdots \\
C A^{\alpha-1}
\end{array}\right]
$$

and

$$
\Sigma_{1} V_{1}^{T}=\left[\begin{array}{llll}
\bar{B}_{1} & A \bar{B}_{1} & \cdots & A^{\beta-1} \bar{B}_{1}
\end{array}\right]
$$

This choice is not unique. Many other choices are also valid. The other common choice is

$$
U_{1} \Sigma_{1}^{1 / 2}=\left[\begin{array}{c}
C \\
C A \\
\vdots \\
C A^{\alpha-1}
\end{array}\right]
$$

and

$$
\Sigma_{1}^{1 / 2} V_{1}^{T}=\left[\begin{array}{llll}
\bar{B} & A \bar{B}_{1} & \cdots & A^{\beta-1} \bar{B}_{1}
\end{array}\right]
$$

Note that the choice of Eq. (22) has the advantage that

$$
U_{1}^{T} U_{1}=I_{n \times n} \Rightarrow U_{1}^{\dagger}=U_{1}^{T}
$$

because $U_{1}$ is a unitary matrix resulting from the property of singular value decomposition. Nevertheless, the choice of Eqs. (24) and (25) has a nice property of 
balanced coordinates. Equation (22) or (24) is commonly called observability matrix whereas Eq. (23) or (25) is referred to as the controllability matrix.

Equations (22) and (23) produce the following solutions

$$
\begin{aligned}
& C=\text { the first } m \text { rows of } U_{1} \\
& \bar{B}_{1}=\text { the first } r \text { columns of } \Sigma_{1} V_{1}^{T}
\end{aligned}
$$

Since the choices of controllability and observability matrices are not unique, the identified matrices $C$ and $\bar{B}_{1}$ are not unique. To determine the state matrix A, let us first define and observe the following matrices.

$$
U_{1 \uparrow}=\left[\begin{array}{c}
C \\
C A \\
\vdots \\
C A^{\alpha-2}
\end{array}\right]
$$

and

$$
U_{\downarrow \downarrow}=\left[\begin{array}{c}
C A \\
C A^{2} \\
\vdots \\
C A^{\alpha-1}
\end{array}\right]=U_{1 \uparrow} A
$$

Deleting the last $m$ rows of $U_{1}$ forms the matrix $U_{1 \uparrow}$ whereas deleting the first $m$ rows of $U_{1}$ yields the matrix $U_{1 \downarrow}$. It is then clear that the state matrix $A$ can be determined by

$$
A=U_{1 \uparrow}^{\dagger} U_{1 \downarrow}
$$

For the identified state matrix to have the rank $n$, both $(\alpha-1) m \times n$ matrices $U_{1 \uparrow}$ and $U_{1 \downarrow}$ must also have the rank $n$. This implies that $\alpha$ must be chosen such that $(\alpha-1) m \geq n$, i.e., $\alpha m>n$. Of course, we have assumed that the pulse force $v_{i}$ for $i=1,2, \ldots, r$ are chosen so that all system modes are excitable and observable.

With the aid of Eq. (13), Eq. (31) produces the continuous-time state matrix as

$$
A_{c}=\frac{1}{\Delta t} \log (A)=\frac{1}{\Delta t} \log \left(U_{1 \uparrow}^{\dagger} U_{1 \downarrow}\right)
$$

Note that the conversion from a discrete-time state matrix to a continuous-time state matrix may not unique. 
To this end, we have determined $A_{c}$ from Eq. (32), $C$ from Eq. (27), $\bar{B}_{1}$ from Eq. (28), and $\bar{D}$ from Eq. (18). The original transmission matrix $\mathrm{D}$ can be recovered using Eq. (9) to have

$$
D=\bar{D} \operatorname{diag}\left[\begin{array}{llll}
1 / v_{1} & 1 / v_{2} & \cdots & 1 / v_{r}
\end{array}\right]
$$

Let us stress that the identified matrices $A_{c}, \bar{B}_{1}$ and $C$ are not uniquely determined but $D$ is coordinate invariant and so is uniquely determined.

\section{Identification of $B_{\mathrm{c}}$, and $N_{\mathrm{ci}}$ :}

The second step begins with generating the two-sample-period pulse response for all inputs with one input at a time, i.e., a force is applied with the same magnitude as above to the system for two time steps $2 \Delta t$. From Eq. (15) for $N=2$, we obtain

$$
\begin{aligned}
& y_{1}(0)=\bar{d}_{1} \quad \ldots \quad y_{r}(0)=\bar{d}_{r} \\
& y_{1}(1)=C \bar{b}_{1}+\bar{d}_{1} \quad \cdots \quad y_{r}(1)=C \bar{b}_{r}+\bar{d}_{r} \\
& y_{1}(2)=C\left[\bar{A}_{1} \bar{b}_{1}+\bar{b}_{1}\right] \quad \cdots \quad y_{r}(2)=C\left[\bar{A}_{r}, \bar{b}_{r}+\bar{b}_{r}\right] \\
& y_{1}(\ell+2)=C A^{\prime}\left[\bar{A}_{1} \bar{b}_{1}+\bar{b}_{1}\right] \quad \cdots \quad y_{r}(\ell+2)=C A^{\prime}\left[\bar{A}_{r} \bar{b}_{r}+\bar{b}_{r}\right]
\end{aligned}
$$

Now define the system Markov parameters from the two-sample-period pulse response as

$$
\begin{aligned}
Y_{2}(2) & =\left[\begin{array}{llll}
y_{1}(2) & y_{2}(2) & \cdots & y_{r}(2)
\end{array}\right] \\
& =\left[\begin{array}{llll}
C\left(\bar{A}_{1} \bar{b}_{1}+\bar{b}_{1}\right) & C\left(\bar{A}_{2} \bar{b}_{2}+\bar{b}_{2}\right) & \cdots & C\left(\bar{A}_{r} \bar{b}_{r}+\bar{b}_{r}\right)
\end{array}\right] \\
Y_{2}(3) & =\left[\begin{array}{llll}
y_{1}(3) & y_{2}(3) & \cdots & y_{r}(3)
\end{array}\right] \\
& =\left[\begin{array}{llll}
C A\left(\bar{A}_{1} \bar{b}_{1}+\bar{b}_{1}\right) & C A\left(\bar{A}_{2} \bar{b}_{2}+\bar{b}_{2}\right) & \cdots & C A\left(\bar{A}_{r} \bar{b}_{r}+\bar{b}_{r}\right)
\end{array}\right] \\
& \vdots \\
Y_{2}(\ell+2) & =\left[\begin{array}{llll}
y_{1}(\ell+2) & y_{2}(\ell+2) & \cdots & y_{r}(\ell+2)
\end{array}\right] \\
& =\left[\begin{array}{llll}
C A^{l}\left(\bar{A}_{1} \bar{b}_{1}+\bar{b}_{1}\right) & C A^{l}\left(\bar{A}_{2} \bar{b}_{2}+\bar{b}_{2}\right) & \cdots & C A^{l}\left(\bar{A}_{r} \bar{b}_{r}+\bar{b}_{r}\right)
\end{array}\right]
\end{aligned}
$$

Subscript 2 for $Y_{2}(k)(k=1,2, \ldots, \ell+1)$ signifies two-sample-period pulse response. Let us form a $\alpha m \times r$ matrix as follows.

$$
H_{2}=\left[\begin{array}{c}
Y_{2}(2) \\
Y_{2}(3) \\
\vdots \\
Y_{2}(\alpha+1)
\end{array}\right]=\left[\begin{array}{c}
C \\
C A \\
\vdots \\
C A^{\alpha-1}
\end{array}\right] \bar{B}_{2}
$$


where

$$
\bar{B}_{2}=\left[\begin{array}{llll}
\left(\bar{A}_{1} \bar{h}_{1}+\bar{b}_{1}\right) & \left(\bar{A}_{2} \bar{b}_{2}+\bar{b}_{2}\right) & \cdots & \left(\bar{A}_{r} \bar{b}_{r}+\bar{b}_{r}\right)
\end{array}\right]
$$

With the help of Eq. (22), the $n \times r$ matrix $\bar{B}_{2}$ in Eq. (36) can be solved by

$$
\bar{B}_{2}=\left[\begin{array}{c}
C \\
C A \\
\vdots \\
C A^{\alpha-1}
\end{array}\right]^{\dagger} H_{2}=U_{1}^{\dagger} H_{2}
$$

Similarly, we may continue the process to generate three-sample-period pulse response, four-sample-period pulse response, etc. up to the $p$-sample-period pulse response for all inputs with one input at a time using a force of the same magnitude as earlier applied to the system for $p$ time periods $p \Delta t$. From Eq. (15) for $N=p$, we have

$$
\begin{array}{cccc}
y_{1}(p)=C\left(\bar{A}_{1}^{p-1} \bar{b}_{1}+\cdots+\bar{b}_{1}\right) & \cdots & y_{r}(p)=C\left(\bar{A}_{r}^{p-1} \bar{b}_{r}+\cdots+\bar{b}_{r}\right) \\
y_{1}(p+1)=C A\left(\bar{A}_{1}^{p-1} \bar{b}_{1}+\cdots+\bar{b}_{1}\right) & \cdots & y_{r}(p+1)=C A\left(\bar{A}_{r}^{p-1} \bar{b}_{r}+\cdots+\bar{b}_{r}\right) \\
y_{1}(p+2)=C A^{2}\left(\bar{A}_{1}^{p-1} \bar{b}_{1}+\cdots+\bar{b}_{1}\right) & \cdots & y_{r}(p+2)=C A^{2}\left(\bar{A}_{r}^{p-1} \bar{b}_{r}+\cdots+\bar{b}_{r}\right) \\
\vdots & \ddots & \vdots \\
y_{1}(p+\ell)=C A^{\prime}\left(\bar{A}_{1}^{p-1} \bar{b}_{1}+\cdots+\bar{b}_{1}\right) & \cdots & y_{r}(p+\emptyset)=C A^{\prime}\left(\bar{A}_{r}^{p-1} \bar{b}_{r}+\cdots+\bar{b}_{r}\right)
\end{array}
$$

Now define the system Markov parameters for the $p$-sample-period pulse response as

$$
\begin{aligned}
& Y_{p}(p)=\left[\begin{array}{llll}
y_{1}(p) & y_{2}(p) & \cdots & y_{r}(p)
\end{array}\right] \\
& \quad=\left[\begin{array}{llll}
C\left(\bar{A}_{1}^{p-1} \bar{b}_{1}+\cdots+\bar{b}_{1}\right) & C\left(\bar{A}_{2}^{p-1} \bar{b}_{2}+\cdots+\bar{b}_{2}\right) & \cdots & C\left(\bar{A}_{r}^{p-1} \bar{b}_{r}+\cdots+\bar{b}_{r}\right)
\end{array}\right] \\
& Y_{p}(p+1)=\left[\begin{array}{llll}
y_{1}(p+1) & y_{2}(p+1) & \cdots & y_{r}(p+1)
\end{array}\right] \\
& \quad=\left[\begin{array}{llll}
C A\left(\bar{A}_{1}^{p-1} \bar{b}_{1}+\cdots+\bar{b}_{1}\right) & C A\left(\bar{A}_{2}^{p-1} \bar{b}_{2}+\cdots+\bar{b}_{2}\right) & \cdots & C A\left(\bar{A}_{r}^{p-1} \bar{b}_{r}+\cdots+\bar{b}_{r}\right)
\end{array}\right] \\
& \quad \vdots \\
& Y_{p}(p+\ell)=\left[\begin{array}{llll}
y_{1}(p+\ell) & y_{2}(p+\ell) & \cdots & y_{r}(p+\ell)
\end{array}\right] \\
& \quad=\left[\begin{array}{llll}
C A^{\prime}\left(\bar{A}_{1}^{p-1} \bar{b}_{1}+\cdots+\bar{b}_{1}\right) & C A^{\prime}\left(\bar{A}_{2}^{p-1} \bar{b}_{2}+\cdots+\bar{b}_{2}\right) & \cdots & C A^{\prime}\left(\bar{A}_{r}^{p-1} \bar{b}_{r}+\cdots+\bar{b}_{r}\right)
\end{array}\right]
\end{aligned}
$$

Let us form a $\alpha m \times r$ matrix as follows.

$$
H_{p}=\left[\begin{array}{c}
Y_{p}(p) \\
Y_{p}(p+1) \\
\vdots \\
Y_{p}(p+\alpha-1)
\end{array}\right]=\left[\begin{array}{c}
C \\
C A \\
\vdots \\
C A^{\alpha-1}
\end{array}\right] \bar{B}_{p}
$$


where

$$
\bar{B}_{p}=\left[\begin{array}{llll}
\left(\bar{A}_{1}^{p-1} \bar{b}_{1}+\cdots+\bar{b}_{1}\right) & \left(\bar{A}_{2}^{p-1} \bar{b}_{2}+\cdots+\bar{b}_{2}\right) & \cdots & \left(\bar{A}_{r}^{p-1} \bar{b}_{r}+\cdots+\bar{b}_{r}\right)
\end{array}\right]
$$

With the help of Eq. (22), the $n \times r$ matrix $\bar{B}_{p}$ in Eq. (41) can be solved by

$$
\bar{B}_{p}=\left[\begin{array}{c}
C \\
C A \\
\vdots \\
C A^{\alpha-1}
\end{array}\right]^{+} H_{p}=U_{1}^{\dagger} H_{p}
$$

To determine $B_{c}$, let us first observe the matrices $\bar{B}_{1}, \bar{B}_{2}, \cdots, \bar{B}_{p}$ defined in Eqs. (20), (37), and (42), and determined by Eqs. (28), (38), and (43), i.e.,

$$
\begin{aligned}
\bar{B}_{1} & =\left[\begin{array}{llll}
\bar{b}_{1} & \bar{b}_{2} & \cdots & \bar{b}_{r}
\end{array}\right] \\
\bar{B}_{2} & =\left[\begin{array}{llll}
\left(\bar{A}_{1} \bar{b}_{1}+\bar{b}_{1}\right) & \left(\bar{A}_{2} \bar{b}_{2}+\bar{b}_{2}\right) & \cdots & \left(\bar{A}_{r} \bar{b}_{r}+\bar{b}_{r}\right)
\end{array}\right] \\
& \vdots \\
\bar{B}_{p} & =\left[\begin{array}{llll}
\left(\bar{A}_{1}^{p-1} \bar{b}_{1}+\cdots+\bar{b}_{1}\right) & \left(\bar{A}_{2}^{p-1} \bar{b}_{2}+\cdots+\bar{b}_{2}\right) & \cdots & \left(\bar{A}_{r}^{p-1} \bar{b}_{r}+\cdots+\bar{b}_{r}\right)
\end{array}\right]
\end{aligned}
$$

Applying the recursive formula

$$
\bar{B}_{k}-\bar{B}_{k-1} ; k=2,3, \cdots, p
$$

yields the controllability-like matrices,

$$
\mathbb{C}_{i}=\left[\begin{array}{llll}
\bar{b}_{i} & \bar{A}_{i} \bar{b}_{i} & \cdots & \bar{A}_{i}^{p-1} \bar{b}_{i}
\end{array}\right] ; i=1,2, \cdots, r
$$

To determine the state matrix $\bar{A}_{i}$, let us first define the two matrices

$$
\mathbb{C}_{i \leftarrow}=\left[\begin{array}{llll}
\bar{b}_{i} & \bar{A}_{i} \bar{b}_{i} & \cdots & \bar{A}_{i}^{p-2} \bar{b}_{i}
\end{array}\right]
$$

and

$$
\mathbb{C}_{i \rightarrow}=\left[\begin{array}{llll}
\bar{A}_{i} \bar{b}_{i} & \bar{A}_{i}^{2} \bar{b}_{i} & \cdots & \bar{A}_{i}^{p-1} \bar{b}_{i}
\end{array}\right]=\bar{A}_{i} \mathbb{C}_{i \leftarrow}
$$

Deleting the last $r$ columns of $\mathbb{C}_{i}$ forms the matrix $\mathbb{C}_{i \leftarrow}$ whereas deleting the first $r$ columns of $\mathbb{C}_{i}$ yields the matrix $\mathbb{C}_{i \rightarrow}$. Equations (47) and (48) produce the solutions:

$$
\begin{aligned}
& \bar{b}_{i}=\text { the first } r \text { columns of } \mathbb{C}_{i} \\
& \bar{A}_{i}=\mathbb{C}_{i \rightarrow} \mathbb{C}_{i \leftarrow}^{+}
\end{aligned}
$$


for $i=1,2, \ldots, r$. For the identified matrix $\bar{A}_{i}$ to have the rank $n$, both $n \times(p-1)$ matrices $\mathbb{C}_{i \leftarrow}$ and $\mathbb{C}_{i \rightarrow}$ must also have the rank $n$. It implies that $p$ must be chosen such that $p-1 \geq n$. This indicates that the system identification method requires a total of at least $(n+1)$ sets of responses generated by $(n+1)$ different time periods of pulse input.

Based on Eqs. (7) and (8) for the definitions of $\bar{A}_{i}$ and $\bar{b}_{i}$, taking the conversion from discrete-time to continuous-time produces

$$
A_{c}+v_{i} N_{c i}=\frac{1}{\Delta t} \log \left(\bar{A}_{i}\right)=\frac{1}{\Delta t} \log \left(\mathbb{C}_{i \rightarrow} \mathbb{C}_{i \leftarrow}^{\dagger}\right)
$$

and

$$
b_{c i}=\frac{1}{v_{i}}\left[\mathrm{I}_{n \times n}+\frac{1}{2 !}\left(A_{c}+\mathrm{v}_{i} N_{c i}\right)(\Delta t)^{2}+\frac{1}{3 !}\left(A_{c}+\mathrm{v}_{i} N_{c i}\right)^{2}(\Delta t)^{3}+\cdots\right]^{-1} \bar{b}_{i}
$$

for $i=1,2, \ldots, r$ where $\mathrm{I}_{n \times n}$ is a $n \times n$ identity matrix, that, in turns, yields

$$
B_{c}=\left[\begin{array}{llll}
b_{c 1} & b_{c 2} & \cdots & b_{c r}
\end{array}\right]
$$

Again, one should be cautious to take the conversion because of its non- uniqueness problem [1]. From Eqs. (32) and (51), the matrices $N_{c i}(i=1,2, \ldots, r)$ are determined by

$$
N_{c i}=\frac{1}{v_{i}}\left[\frac{1}{\Delta t} \log \left(\bar{A}_{i}\right)-A_{c}\right]=\frac{1}{v_{i} \Delta t}\left[\log \left(\mathbb{C}_{i \rightarrow} \mathbb{C}_{i \leftarrow}^{+}\right)-\log \left(U_{1 \uparrow}^{+} U_{1 \downarrow}\right)\right]
$$

To this end, we have identified all continuous-time system matrices $A_{\mathrm{c}}, B_{\mathrm{c}}, N_{\mathrm{ci}}, C$, and $D$ for the bilinear system described by Eqs. (1) and (2) from pulse responses generated by pre-specified pulse inputs.

\section{Coordinate Transformation}

Let the state vector $x$ of $n \times 1$ in Eqs. (1) and (2) be transformed to the new state vector $\tilde{x}$ of $n \times 1$ by the nonsingular transformation matrix $\Phi$ of $n \times n$. Equations (1) and (2) become

$$
\dot{\tilde{x}}=\tilde{A}_{c} \tilde{x}+\tilde{B}_{c} u+\sum_{i=1}^{r} \tilde{N}_{c i} \tilde{x} u_{i}
$$

and

$$
y=\tilde{C} \tilde{x}+D u
$$

where 


$$
\begin{aligned}
& \tilde{x}=\Phi^{-1} x \\
& \tilde{A}_{c}=\Phi^{-1} A_{c} \Phi \\
& \tilde{N}_{c i}=\Phi^{-1} N_{c i} \Phi \\
& \tilde{B}_{c}=\Phi^{-1} B_{c} \\
& \tilde{C}=C \Phi
\end{aligned}
$$

It is clear that the transformed matrix $\tilde{A}_{c}$ is similar to the original matrix $A_{c}$ in the sense that their eigenvalues are identical. The same statement is also true for the matrices $\tilde{N}_{c i}$ and $N_{c i}$. One question that may arise is, for given two sets of matrices representing the same bilinear system, what the transformation matrix should be to convert from one coordinate to the other.

First, form the observability matrices for both sets of system matrices

$$
Q=\left[\begin{array}{c}
C \\
C A_{c} \\
\vdots \\
C A_{c}^{n-1}
\end{array}\right]
$$

and

$$
\tilde{Q}=\left[\begin{array}{c}
\tilde{C} \\
\tilde{C} \tilde{A}_{c} \\
\vdots \\
\tilde{C} \tilde{A}_{c}^{n-1}
\end{array}\right]
$$

Substituting the relationship from Eq. (57) yields

$$
\tilde{Q}=\left[\begin{array}{c}
\tilde{C} \\
\tilde{C} \tilde{A}_{c} \\
\vdots \\
\tilde{C} \tilde{A}_{c}^{n-1}
\end{array}\right]=\left[\begin{array}{c}
C \\
C A_{c} \\
\vdots \\
C A_{c}^{n-1}
\end{array}\right] \Phi=Q \Phi
$$

that in turn provide the following solution for computing the transformation matrix $\Phi$

$$
\Phi=Q^{\dagger} \tilde{Q}
$$


This transformation matrix $\Phi$ will transform the original system coordinate to another system coordinate without changing the input-to-output map.

\section{Numerical Example}

Consider the following example presented in Ref. [4]

$$
\begin{aligned}
& \dot{x}=A_{c} x+B_{c} u+N_{c 1} x u_{1}+N_{c 2} x u_{2} \\
& y=C x
\end{aligned}
$$

where

$$
\begin{aligned}
& A_{c}=\left[\begin{array}{cc}
-1 & 0 \\
1 & -2
\end{array}\right] ; \quad N_{c 1}=\left[\begin{array}{ll}
0 & 0 \\
1 & 1
\end{array}\right] ; N_{c \cdot 2}=\left[\begin{array}{ll}
1 & 1 \\
0 & 0
\end{array}\right] \\
& B_{c}=\left[\begin{array}{ll}
1 & 0 \\
0 & 1
\end{array}\right] ; \quad C=\left[\begin{array}{ll}
0 & 1
\end{array}\right]
\end{aligned}
$$

Assume that we don't know the order of the system. Let us generate five sets of data with the time period $\Delta t=1$ second. The first set of data with one input at a time is generated by a unit force of period 1 second. The second set of data with one input at a time is obtained by applying a unit force of period 2 seconds and the fifth set of data is computed with a unit force of period 5 seconds. The total of data points is set to be 20 for each data record.

Figure 1 shows a total of $10(p \times m \times r)$ responses from 2 inputs $(r=2), 1$ output $(m=1)$, and 5 different multiple-pulse inputs $(p=5)$. Each response sampled at $1 \mathrm{~Hz}$ has 20 data points. These ten responses are obtained by numerically integrating the bilinear system shown in Eq. (62). With $\alpha=5$ and $\beta=6$, the Hankel matrix $H_{1}$ shown in Eq. (19) should have the size of $5 \times 12(\alpha m \times \beta r)$. The state matrix and the output matrix identified from this Hankel matrix is

$$
\tilde{A}_{c}=\left[\begin{array}{cc}
-1.0629 & 3.9782 \\
0.0148 & -1.9371
\end{array}\right] ; \quad \tilde{C}=\left[\begin{array}{ll}
-0.9355 & 0.3497
\end{array}\right]
$$

The singular values of this Hankel matrix are

$$
\Sigma_{1}=\operatorname{diag}\left[\begin{array}{lllll}
0.8347 & 0.0543 & 0 & 0 & 0
\end{array}\right]
$$

implying that the order of the system is $n=2$. The other matrices $H_{\mathrm{k}}$ for $\mathrm{k}=2,3, \ldots, p$ shown in Eqs. (36) and (41) are of the size of $5 \times 2$, that produce the matrices 
$\bar{B}_{1}, \bar{B}_{2}, \cdots, \bar{B}_{5}$ of $2 \times 2$ each shown in Eq. (44), and in turn yield $\mathbb{C}_{1}$ and $\mathbb{C}_{2}$ of $2 \times 5$ each shown in Eq. (47). The $B_{\mathrm{c}}, N_{\mathrm{c} 1}$, and $N_{\mathrm{c} 2}$ can thus be identified as

$$
\begin{aligned}
\tilde{N}_{c 1} & =\left[\begin{array}{rr}
1.7752 & 3.2911 \\
-0.4182 & -0.7752
\end{array}\right] ; \quad \tilde{N}_{c 2}=\left[\begin{array}{ll}
0.1678 & 0.3111 \\
0.4489 & 0.8322
\end{array}\right] \\
\tilde{B}_{c} & =\left[\begin{array}{rr}
-0.0929 & -0.9824 \\
-0.2484 & 0.2314
\end{array}\right]
\end{aligned}
$$

The quality of the identification of the system is evaluated next. The following transformation matrix is computed from Eq. (61)

$$
\Phi=\left[\begin{array}{rr}
-0.8715 & -3.6998 \\
-0.9355 & 0.3497
\end{array}\right]
$$

the matrices $\tilde{A}_{c}, \tilde{B}_{c}, \tilde{C}_{,} \tilde{N}_{c 1}$ and $\tilde{N}_{c 2}$ would be transformed by using Eq. (57) to become $A_{\mathrm{c}}, B_{\mathrm{c}}, C, N_{\mathrm{cl}}$, and $N_{\mathrm{c} 2}$ shown in Eq. (63).

Let us examine another case where we keep the unit force for the first input but change to 0.5 for the second input. Figure 2 shows the 10 multiple-pulse responses. Applying the same identification procedure as above, we obtain the following system matrices

$$
\tilde{A}_{c}=\left[\begin{array}{cc}
-0.9509 & 3.9503 \\
0.0131 & -2.094
\end{array}\right] ; \quad \tilde{C}=\left[\begin{array}{ll}
-0.9253 & 0.3760
\end{array}\right]
$$

and

$$
\begin{aligned}
\tilde{N}_{c 1} & =\left[\begin{array}{rr}
1.8543 & 3.2168 \\
-0.4925 & -0.8543
\end{array}\right] ; \quad \tilde{N}_{c \cdot 2}=\left[\begin{array}{ll}
0.1898 & 0.3292 \\
0.4670 & 0.8102
\end{array}\right] \\
\tilde{B}_{c} & =\left[\begin{array}{rr}
-0.0998 & -0.9755 \\
-0.2457 & 0.2591
\end{array}\right]
\end{aligned}
$$

with the transformation matrix

$$
\Phi=\left[\begin{array}{rr}
-0.9757 & -3.6737 \\
-0.9253 & 0.3760
\end{array}\right]
$$

Note that the Hankel singular values are

$$
\Sigma_{1}=\operatorname{diag}\left[\begin{array}{lllll}
0.6753 & 0.0489 & 0 & 0 & 0
\end{array}\right]
$$

This set of system matrices also represents the bilinear system, because it can be transformed back to the original system exactly. 


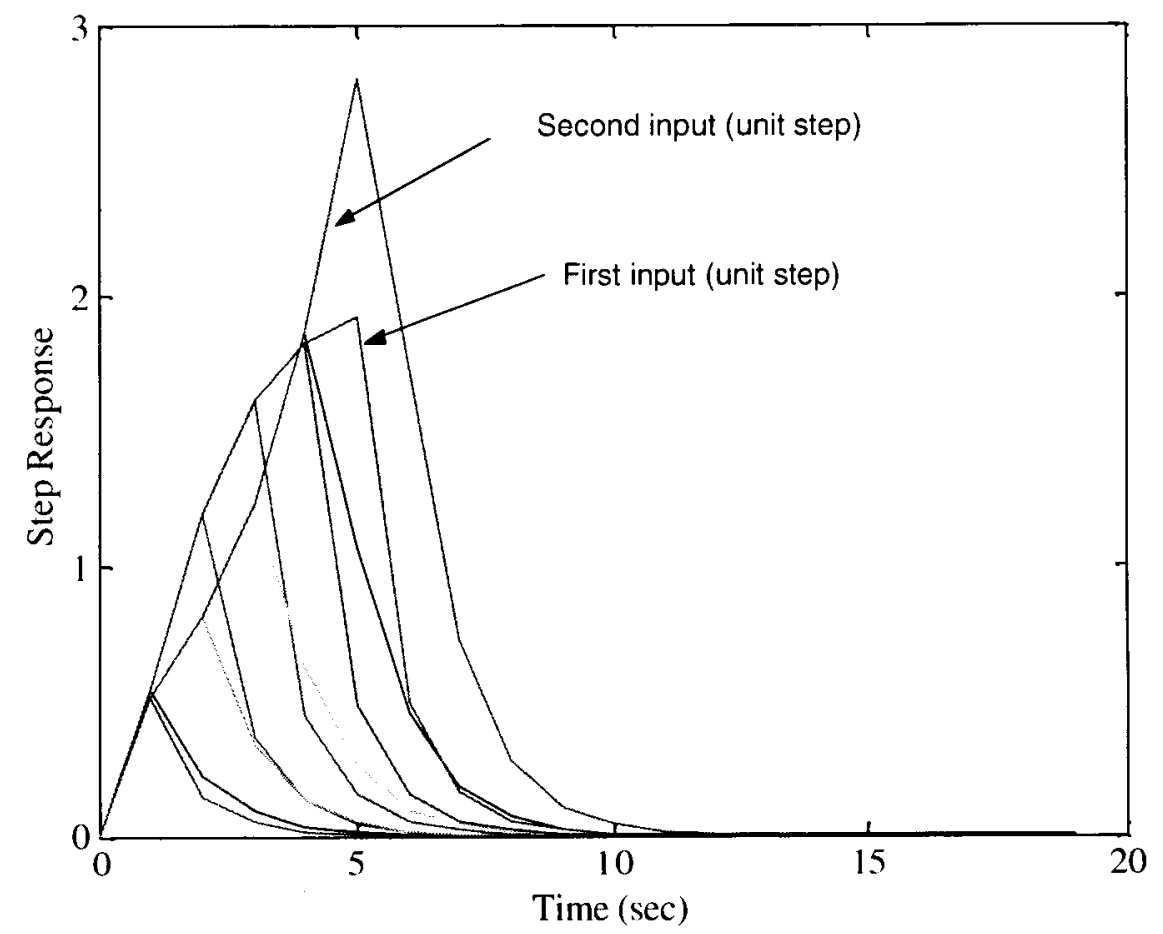

Figure 1: Five sets of pulse responses sampled at $1 \mathrm{~Hz}$ from two inputs of unit pulse.

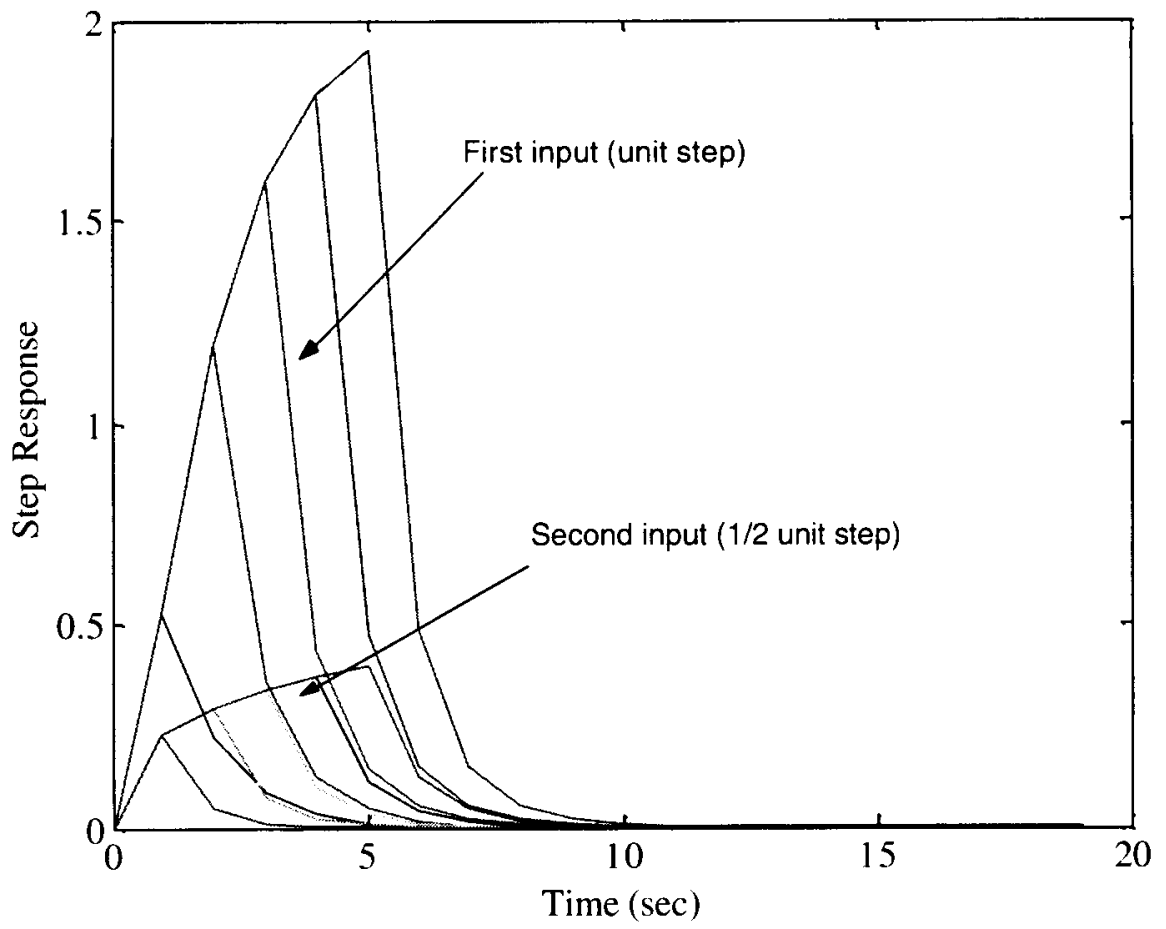

Figure 2: Five sets of pulse responses sampled at $1 \mathrm{~Hz}$ from two different-size inputs. 


\section{Concluding Remarks}

A new method is introduced for identification of a continuous-time multi-input and multi-output bilinear system. The approach is to make judicious use of the linearmodel properties of the bilinear system when subjected to a constant input. It has been shown in this paper that a bilinear system can be treated as a combination of two linear systems in the identification process. The first linear system is the one obtained by deleting the nonlinear terms of the bilinear system. The second linear system is given by assuming a constant input. Due to this latter property, the identification process for the bilinear system becomes a combination of two linear-system identification processes. The key is to combine these two linear-system identification processes in the same coordinate system. The resulting identified system matrices would be similar to the original ones in the sense that they represent the same bilinear system but in different coordinates. With a proper coordinate transformation, both the original model and the identified model are identical.

\section{References}

1. Juang, J.-N., Applied System Identification, Prentice Hall, Inc., Englewood Cliffs, New Jersey 07632, 1994, ISBN 0-13-079211-X.

2. Juang, J-N. and Phan, M. Q., Identification and Control of Mechanical Systems, Cambridge University Press, New York, NY 10011-4211, 2001, ISBN 0521-78355-0.

3. Natke, H. G., Juang, J. N., and Gawronski, W., "A Brief Review on the Identification of Nonlinear Mechanical Systems," Proceedings of the 6th International Modal Analysis Conference, Orlando, Florida, Feb. 1-4, 1988, pp. 1569-1574.

4. Bruni, C., Pillo, G. Di., Koch, G., "On the Mathematical Models of Bilinear Systems," Ricerche Di Automatica, Vol. 2, No.1, January 1971. 
5. Bruni, C., Pillo, G. Di., Koch, G., "Bilinear Systems: An Appealing Class of Nearly Linear Systems in Theory And Application," IEEE Trans. Automat. Control, AC19:334-348, August 1974.

6. Mohler, R. R., and Kolodziej, “An Overview of Bilinear System Theory and Applications," IEEE Transactions on Systems, Man and Cybernetics, SMC-10, pp. $683-688,1980$

7. Mohler, R. R., Nonlinear Systems: Vol. I, Dynamics and Control, Vol.II, Applications to Bilinear Control, Prentice-Hall, Englewood Cliffs, New Jersey, 1991

8. Elliott, D. L., Bilinear Systems, Encyclopedia of Electrical Engineering, Edited by John Webster, J. Wiley and Sons, 1999.

9. Rangarathan, V., Jha, A.N., and Rajamani, V.S., "Recursive Estimation Algorithms for Bilinear and Nonlinear Systems Using a Polynomial Approach," International Journal of Control, Vol. 44, No. 2, 1986, pp. 419-426.

10. Billings, S. A. and Voon, W. S. F., "Piecewise Linear Identification of Non-Linear Systems, “International Journal of Control, Vol. 46, No. 1, 1987, pp. 215-235.

11 Dai, H. and Sinha, N. K., "Robust Identification Of Systems Using Block-Pulse Functions," Control Theory and Applications, IEE Proceedings Part D, Vol. 139, No. 3, 1992, pp. 308-316.

12. Ralston, J. C., and Boashash, B., "Identification of Bilinear System Using Bandlimited Regression, Proceedings of the 1997 IEEE International Conference on Acoustics, Speech, and Signal Processing, Munich, Germany, April 1997, pp. 39253928.

13. Banerjee, A., Arkun, Y., Ogunnaike, B., and Pearson, R., "Estimation of Nonlinear Systems Using Linear Multiple Models," AIChE Journal 43(5), 1997, pp.1204-1226.

14 Dunoyer, A., Balmer, L., Burnham, K. J., and James, D. J. G., "On the Discretization of Single-Input Single-Output Bilinear Systems," International Journal of Control, Vol. 68, No. 2,1997, pp. 361-372. 
15. Brewer, J., "Bilinear Equations in Ecology," Systems and Control Encyclopedia: Theory, Technology, Applications, Madan G. Singh (Ed.), Pergamon Press, Oxford, Volume 1, 1987, pp. 414-417.

16 Daniel-Berhe, S. and Unbehauen, H., (1998a), "Bilinear Continuous-Time Systems Identification via Hartley-Based Modulating Functions," Automatica, Vol. 34, No. 4, 1998, pp. 499-503.

17 Daniel-Berhe, S. and Unbehauen, H., "State Space Identification of Bilinear Continuous-Time Canonical Systems via Batch Scheme Hartley Modulating Functions Approach," Proceedings of the 37th IEEE Conference on Decision and Control, Tampa, Florida (December), 1998, pp. 4482-4487.

18. Boukhris, A., Mourot, G., and Ragot, J., "Non-Linear Dynamic System Identification: A Multi-Model Approach," International Journal of Control, Vol. 72, No. 7/8, 1999, pp. 591-604.

19. Verdult, V., "Nonlinear System Identification: A State-Space Approach," Twente University Press, Netherlands, Ph. D. thesis, 2002, ISBN 90-365-1717-6. 


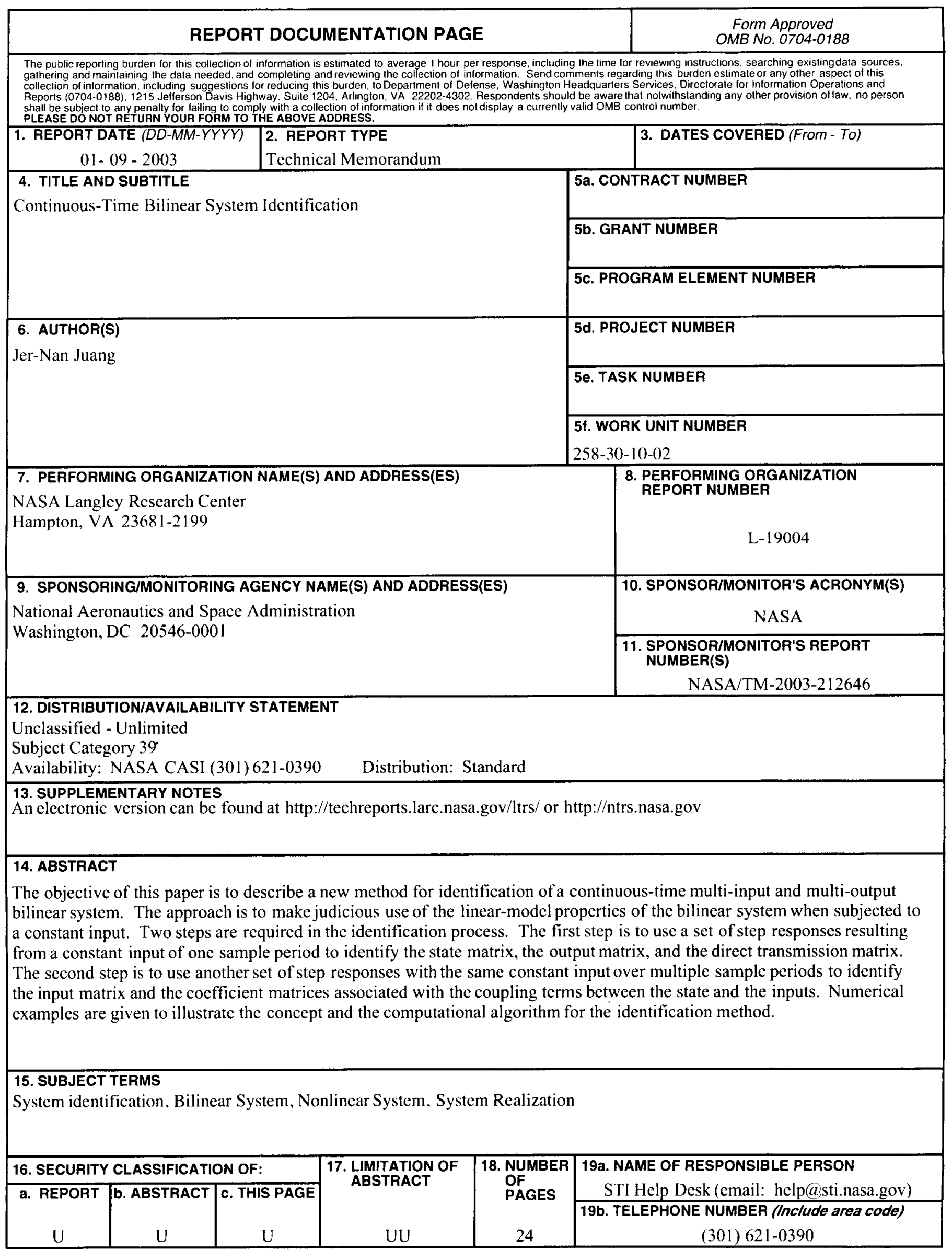

